\title{
Interactions between auditory statistics processing and visual experience emerge only in late development
}

\author{
Martina Berto ${ }^{1}$, Pietro Pietrini ${ }^{1}$, Emiliano Ricciardi ${ }^{1}$, Davide Bottari ${ }^{1,}$ \\ ${ }^{1}$ Molecular Mind Lab, IMT School for Advanced Studies, Lucca, 55100, Italy \\ ${ }^{*}$ Corresponding author: Davide Bottari \\ Email: davide.bottari@imtlucca.it
}

Author Contributions: Conceptualization, Davide Bottari and Martina Berto; Methodology, Martina Berto and Davide Bottari; Investigation Martina Berto and Davide Bottari; Writing - Original Draft, Martina Berto and Davide Bottari; Review and Editing, Martina Berto, Davide Bottari, Emiliano Ricciardi, and Pietro Pietrini; Data visualization, Martina Berto and Davide Bottari.

Competing Interest Statement: The authors declare no competing interests.

Keywords: Computational auditory neuroscience; Audio-Visual interplay; Development of basic auditory computations; Visual deprivation. 


\section{ABSTRACT}

3 The human auditory system relies on both detailed and summarized representations to recognize

4 different sounds. As local features can exceed the storage capacity, average statistics are computed over

5 time to generate more compact representations at the expense of temporal details availability. This study

6 aimed to identify whether these fundamental sound analyses develop and function exclusively under the

7 influence of the auditory system or interact with other modalities, such as vision. We employed a

8 validated computational synthesis approach allowing to control directly statistical properties embedded in

9 sounds. To address whether the two modes of auditory representation (local features processing and

10 statistical averaging) are influenced by the availability of visual input in different phases of development,

11 we tested samples of sighted controls (SC), congenitally blind (CB), and late-onset (> 10 years of age)

12 blind (LB) individuals in two separate experiments which uncovered auditory statistics computations from

13 behavioral performances. In experiment 1, performance relied on the availability of local features at

14 specific time points; in experiment 2, performance benefited from computing average statistics over

15 longer durations. As expected, when sound duration increased, detailed representation gave way to

16 summary statistics in SC. In both experiments, the sample of CB individuals displayed a remarkably

17 similar performance revealing that both local and global auditory processes are not altered by blindness

18 since birth. Conversely, LB individuals performed poorly compared to the other groups when relying on

19 local features, with no impact on statistical averaging. The dampening in the performance was not

20 associated with the onset and duration of visual deprivation. Results provide clear evidence that vision is

21 not necessary for the development of the auditory computations tested here. Remarkably, a functional

22 interplay between acoustic details processing and vision emerges at later developmental phases.

23 Findings are consistent with a model in which the efficiency of local auditory processing is vulnerable in

24 case sight becomes unavailable. Ultimately results are in favor of a shared computational framework for

25 auditory and visual processing of local features, which emerges in late development. 


\section{INTRODUCTION}

33 The auditory system is specialized in capturing fine-grained details from sound waves (Plomp, 1964).

34 These local temporal features are then integrated over time by mechanisms that retain, summarize, and abstract them into more compact acoustic percepts (Yabe et al., 1998; McDermott, Schemitsch, and Simoncelli, 2013). Computational synthesis approaches, derived from information theories (Barlow, 1961), allowed to investigate these processes by implementing mathematical models to describe the set of measurements that the auditory system operates (McDermott and Simoncelli, 2011; McDermott, Schemitsch, and Simoncelli, 2013). Such models have revealed that stationary sounds are analyzed by extracting a set of auditory statistics (McDermott and Simoncelli, 2011) whose processing represents the keystone of acoustic features integration. Auditory statistics result from a set of computations strictly dependent on anatomical and functional properties of the auditory pathway (Ruggero, 1992; Dau, Kollmeier and Kohlrausch, 1997; Gygi, Kidd and Watson, 2004; Joris, Schreiner and Rees, 2004; Baumann et al., 2011), and can be consequently described only through biologically plausible models (McDermott and Simoncelli, 2011). The auditory system processes these statistics by averaging shortterm acoustic events (McDermott and Simoncelli, 2011; McDermott, Schemitsch, and Simoncelli, 2013; McWalter and McDermott, 2018), along specific time windows (McWalter and McDermott, 2018) and uses this information to derive compact representations of sound objects. The auditory statistics processing can be broken down into two main modes of representation. (1) Local features processing, by which finegrained temporal details are extracted from a sound and stored; (2) Statistical averaging, by which local features are averaged within a time-window of integration, resulting in a global representation as local details are no longer retained (McDermott, Schemitsch, and Simoncelli, 2013).

While auditory statistics represent unimodal computations, the auditory system does not develop and function in isolation, and other senses, such as vision, modulate its functional and structural organization. Studies in non-human animal models revealed that, in the early development, the onset of visual input (eyes opening) gates the critical period closure of basic auditory functions. This evidence suggested that visually modulated sensitive periods in the auditory cortex exist (Mowery, Kotak, and Sanes, 2016). In

58 adults, visual events are known to directly modulate auditory neuron responses in both animals (Kayser, 59 Petkov, and Logothetis, 2008) and humans (Thorne et al., 2011). At a functional level, visual systems

60 play an important role in auditory features segregation, helping in disambiguating difficult instances (e.g., 61 Golumbic et al., 2013). A common computational framework for feature extraction might exist between 62 auditory and visual modality (Shamma, 2001). In the same vein, the set of auditory statistics evaluated 63 here were derived by auditory computational models (McDermott and Simoncelli, 2011), which are 64 conceptually very similar to those derived by visual ones (Portilla and Simoncelli, 2000). In the present 65 study, we directly investigated if the auditory statistics development and functioning fall within the exclusive competence of the auditory systems, or are instead influenced by vision. 
67 Visual deprivation models have been systematically employed to indirectly uncover the audio-visual 68 interplay (for review, see Röder, Kekunnaya, and Guerreiro, 2020). The lack of visual inputs has 69 consistently been associated with altered auditory processing across different functions (for review, see

70 Röder and Pavani, 2012), but whether visual input availability alters specific underlying auditory 71 computations is still unknown. In humans, previous evidence has identified visual inputs availability since 72 birth as a prerequisite for the full development of auditory spatial calibration (Gori et al., 2014). On a 73 different note, a large body of evidence suggests that both congenital and late-onset visual deprivation 74 exert a compensating influence over certain higher-order auditory functions (see Röder, Kekunnaya, and 75 Guerreiro, 2020). Compensatory effects have been consistently observed both in the context of spatial 76 processing of auditory stimuli (e.g., Battal et al., 2019) and in tasks requiring spectro-temporal auditory 77 features analyses, such as mnemonic representations of sounds (Röder and Rösler 2003), verbal 78 memory (Amedi et al., 2003), frequency tuning (Huber 2019), speech comprehension (Trouvain, 2007; 79 Dietrich, Hertrich, and Ackermann, 2013), and auditory temporal resolution (Muchnik et al., 1991).

80 To address whether the two modes of auditory representation, the local features processing and 81 statistical averaging, are differently influenced by visual input availability, we tested blind populations 82 against sighted individuals in two experiments designed to tap into these two computational modes. By 83 combining a sound synthesis algorithm (McDermott and Simoncelli, 2011; McDermott, Schemitsch, and 84 Simoncelli, 2013) with psychophysics, the methodology adopted here provided the opportunity to uncover 85 local and global auditory statistics computations from the discriminative responses elicited by different 86 sound properties. Importantly, comparing samples of individuals who were visually deprived since birth or 87 in late phases of development allowed to assess whether the selected aspect of sound representation 88 interacts with vision at specific time points along the life span.

89 We could expect detrimental behavioral effects associated with the lack of vision (e.g., Gori et al., 2014) 90 in one of the samples of blind individuals or both. This outcome would syndicate for a modality interplay 91 between vision and auditory computations in which vision plays a crucial role in supporting specific 92 aspects of basic auditory processing (i.e., acoustic features segregation; Park et al., 2016). Noticeably, 93 some auditory fundamentals, such as periodicity pitch, are innate and not influenced by early experience 94 (Montgomery and Clarkson, 1997). Thus, sensory experience might also not be required for the full 95 development of the functions tested here, but modality interplays could still occur later. However, 96 provided that several auditory processes can benefit from the lack of vision, behavioral compensatory 97 effects in one or both visual deprivation models (congenital and late-onset blindness) could also be 98 expected in our study. Such results would indicate that vision is not necessary for their development or 99 functioning and would provide evidence that a functional adaptation to lack of vision occurs not only for 100 high-order functions but also for selective basic auditory computations. In both scenarios, a difference 101 between CB and LB group would characterize the developmental trajectory of audio-visual interplay in the 102 context of local and global auditory statistics processing. 
We took advantage of an already corroborated methodological pipeline (McDermott, Schemitsch, and Simoncelli, 2013), and produced different synthetic textures built upon original recordings. Thanks to their properties, a specific category of natural sounds, namely Sound Textures, was used (some examples are the rain, fire, bulldozer, typewriting, waterfall sounds; McDermott \& Simoncelli, 2011; Saint-Arnaud \& Popat, 2006; Schwarz, 2011). These sounds are rich, ubiquitous, and constant over time (McDermott, Schemitsch, and Simoncelli, 2013; McWalter and McDermott, 2018). We selected 54 environmental recordings of Sound Textures (Table S1, Supplementary Information), among the original set implemented by McDermott, Schemitsch, and Simoncelli, 2013. Synthetic stimuli were created using an Auditory Texture Model (McDermott and Simoncelli, 2011), which efficiently simulates computations performed at peripheral stages of the auditory processing. By computing time-averages of non-linear functions, it was possible to measure a set of statistics: envelope marginal moments, reflecting distribution and sparsity of the signal (Lorenzi et al., 1999), envelope cross-correlation between cochlear envelopes, accounting for the presence of broadband events within the signal (McDermott and Simoncelli, 2011), the modulation bands power, providing information about the temporal structure within cochlear channels (Bacon and Wesley Grantham, 1989; Dau, Kollmeier and Kohlrausch, 1997; McDermott and Simoncelli, 2011), and their correlations (McDermott and Simoncelli, 2011). Although the model represents a mathematical approximation, cochlear envelope statistics convey most of the perceptually relevant information about the sound (Smith, Delgutte and Oxenham, 2002; Gygi, Kidd and Watson, 2004; McDermott and Simoncelli, 2011). By using the full set of statistics to generate synthetic sounds, it is possible to obtain compelling exemplars of the same original Sound Texture (McDermott and Simoncelli, 2011). Our experimental stimuli were created accordingly. By imposing the statistics mentioned above on four different white noise samples, we synthesized four different exemplars for each original Sound Texture. Among themselves, synthetic exemplars of the same texture varied only for their local features, while their long-term average statistics matched the sound they were derived from. In other terms, this process resulted in four synthetic sounds whose properties were constrained only by the selected average statistics extracted from the original recording (Figure 1A). This provided us with the unique opportunity to test for specific computations within the auditory statistics processing, thanks to the

133 unparalleled level of control exerted over the statistics present in the synthesized sounds (for details 134 about synthesis procedure, see Materials and Methods).

135 We tested sighted and blind participants in two selected experiments which included our synthetic sounds 136 and exploited the two modes of auditory statistics representation.

137 Blind participants were grouped according to blindness onset, whether from birth or developmentally later 138 (>10 years old; see Table 1). Thus, three groups of participants were recruited: congenitally blind (CB), 139 late-onset blind (LB), and sighted control (SC) individuals. All three groups were matched by sample size, 140 age, and gender (see Materials and Methods). 
141 Both experiments consisted of a two-alternative forced-choice oddity (2AFC); participants had to detect

142 the deviant sound among three acoustic samples, selecting between first and third intervals. Stimuli were

143 created by cutting each synthetic exemplar into smaller excerpts of different lengths. Each trial included

144 three excerpts of the same length. The duration of the excerpts varied across trials, for a total of six

145 durations (McDermott, Schemitsch, and Simoncelli, 2013).

146 In the first experiment, Exemplar Discrimination, participants were asked to report which sound (the first

147 or the third) was different from the other two. Two excerpts were the exact same sound. By contrast, the

148 odd one was an excerpt extracted from a different synthetic exemplar of the same Sound Texture (Figure

149 1B). Thus, all of them stemmed from the same sound source (e.g., bulldozer), but one was generated

150 starting from a different white noise and consistently diverged for the local features it encompassed as

151 compared to the other two. Nonetheless, as duration increased, average statistics tended to converge

152 towards the original imposed values (Figure 1D, left panel), making all three sounds perceptually very

153 similar and challenging task performance.

154 In the second experiment, Texture Discrimination, participants were asked to report which sound came 155 from a different acoustic source. Two excerpts were extracted from two distinct synthetic exemplars of the 156 same Sound Texture (e.g., bulldozer), while the deviant was drawn from a synthetic exemplar derived 157 from a different one (e.g., waterfall). Therefore, only the deviant in the triplet comprised both different 158 local features and imposed average statistics (Figure 1C). Since all three sounds represented different 159 excerpts, the local variability between couples was never zero. Still, the average statistics variability 160 between the two sounds originated from the same texture tended to progressively decrease with duration, 161 allowing for the different sound source (the deviant) to emerge perceptually (Figure 1D, right panel). 
A

\section{Schematic representation of Sound Texture Synthesis}

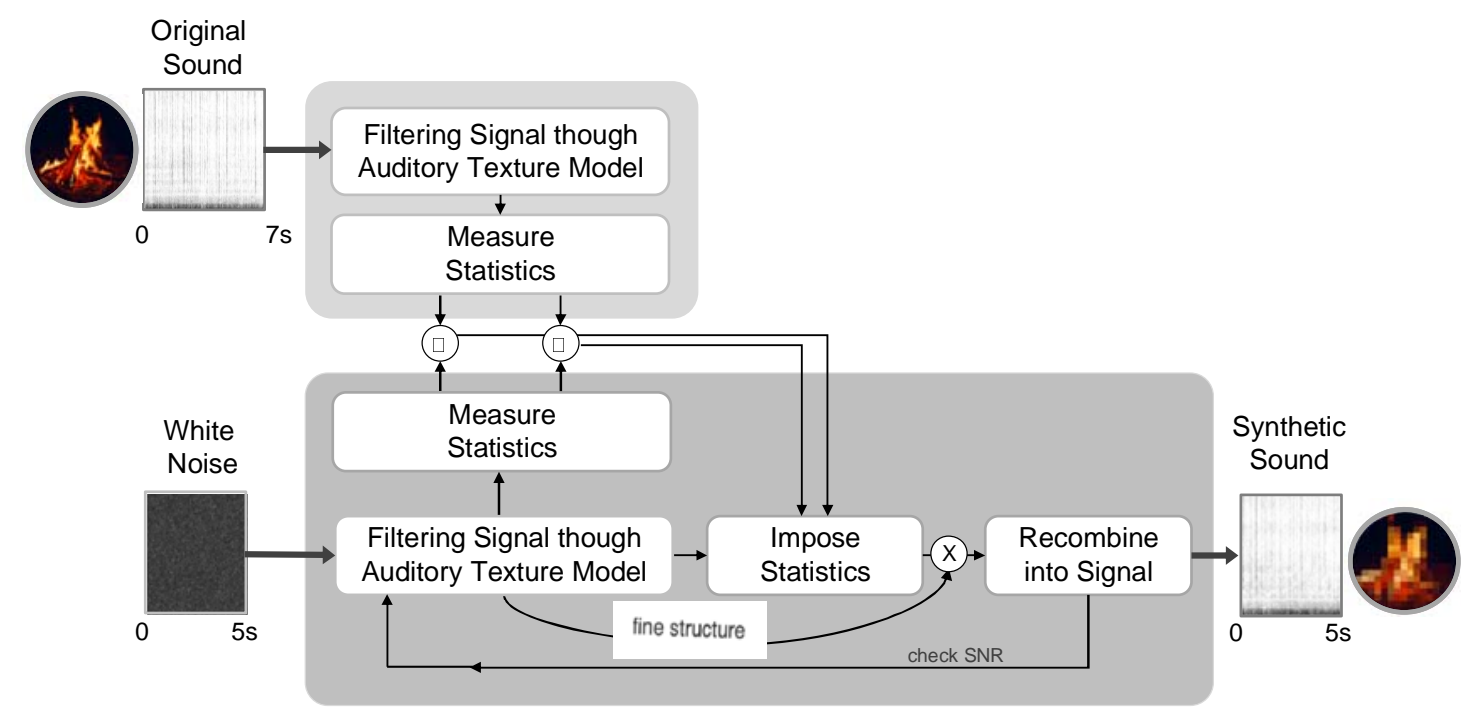

B

\section{Exemplar Discrimination}

Which sound is different from the others? First or last? Use the middle one as a reference

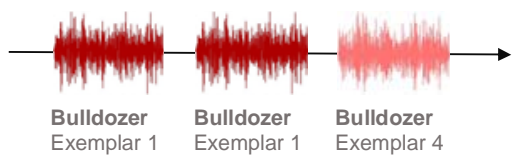

\section{C}

\section{Texture Discrimination}

Which sound comes from a different source? First or last? Use the middle one as a reference

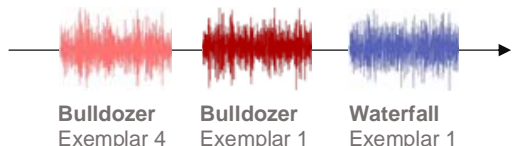

D

Average statistical variability among sound triplets in each experiment
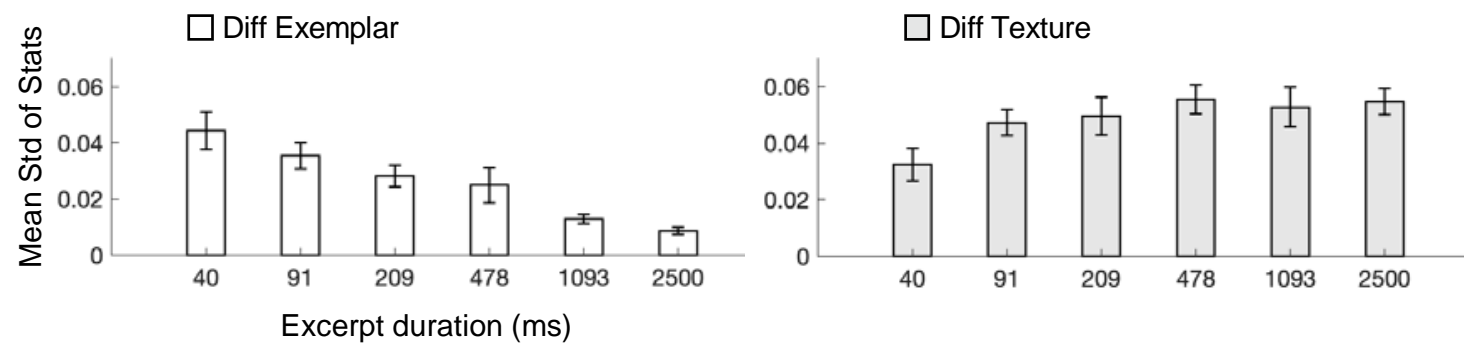

Figure 1. Sound Texture Synthesis, Auditory Statistics Variability, and Experimental Design.

(A) Schematic representation of the Sound Texture Synthesis procedure employed to produce synthetic stimuli. A 7-s Original Recording was passed through the Auditory Texture Model (McDermott and Simoncelli, 2011) to extract average statistics of interest. A white noise sample was then passed again through the model while the Original Recording statistics were imposed on its cochlear envelopes. Modified envelopes were multiplied by their associated fine structure and recombined into the signal. This procedure was iterated until a desirable signal-to-noise ratio is reached. The outcome was a Synthetic Sound Texture Exemplar, a 5-s signal containing statistics that matched the original values and was only constrained by them. Adapted from McDermott and Simoncelli, 2011.

(B) Exemplar Discrimination. Schematic representation of one trial. Participants had to detect the different sound among the three, being the other two identical. In this case, the correct answer is the last sound since it is another exemplar of the "Bulldozer" Sound Texture. 
(C) Texture Discrimination. Schematic representation of one trial. Participants had to report which sound was coming from a different source with respect to the other two. In this example, the correct answer is the third sound, being it an excerpt of a different Sound Texture

(D) Average variability across a set of statistics (envelope mean, skewness, variance, cross-correlation and modulation power) was computed from couples of excerpts, to measure, in both experiments, the objective statistical difference between reference and deviant sounds. Left: average standard deviation (std) across statistics measured in excerpt pairs originated from Different Exemplars of the same Sound Texture (for all textures in column 1, Table S1, Supplementary Information). When duration increased, average statistics converged to the imposed original values, and variability progressively tended to zero, increasing discrimination difficulty in Exemplar Discrimination. Right: average std across statistics measured in excerpts pairs derived from Different Sound Textures (columns 1 and 2, Table S1, Supplementary Information). In Texture Discrimination, both scenarios (Different Exemplar and Different Texture) were presented and compared against each other. As duration increased, the difference between the two contexts increased, facilitating the recognition of the deviant one.

(See also Figure S1 and Table S1)

The employment of these experiments allowed for specific predictions on the outcomes. Consistent with previous findings by McDermott, Schemitsch, and Simoncelli (2013), opposite patterns of results were expected in the two experiments as a function of excerpts duration. For short stimuli, due to differences in features variability among the three sounds, good performance was expected in Exemplar Discrimination compared to Texture Discrimination. By contrast, for long stimuli, statistical averaging was expected to boost Texture Discrimination performance and to hamper Exemplar Discrimination one (McDermott,

184 Schemitsch, and Simoncelli, 2013). Any significant deviation from these expected outcomes indicates 185 changes in the processing of local features or statistical averaging.

186 The attended pattern of results (McDermott, Schemitsch, and Simoncelli, 2013) was confirmed in our SC group. The performance was better for short durations $(40,91 \mathrm{~ms})$ in Exemplar Discrimination as compared to Texture Discrimination (all $p<0.003$, corrected). Conversely, for long durations $(478,1093$, $2500 \mathrm{~ms}$ ), participants' accuracy was higher in Texture Discrimination as compared to Exemplar Discrimination (all $p<0.03$, corrected). No difference was observed for trials comprising stimuli that were $209 \mathrm{~ms}$ long ( $\mathrm{p}=0.31$, corrected; Figure $2 \mathrm{~A}$ ). Overall, the data replicated previous findings in sighted individuals, despite participants being blindfolded. Thus, these results represent a validated context to assess whether visual experience impacts auditory statistics processing.

The CB group displayed a remarkably similar pattern of results. Better performance was found in Exemplar Discrimination for short durations (40, 91ms) compared to Texture Discrimination (all $p<0.02$,

197 Exemplar (all $p<0.006$, corrected). As observed in SC, there was no difference between the two 198 experiments for stimuli that were $209 \mathrm{~ms}$ long $(p=0.86$, corrected; Figure $2 B)$.

199 By contrast, in the LB group, no significant differences were found between Exemplar Discrimination and 200 Texture Discrimination for all short stimuli (40, 91, 209ms; all $p>0.05$, corrected). However, for long 201 durations (478, 1093, 2500ms), LB participants performed better in Texture Discrimination as compared 
to Exemplar Discrimination (all adjusted $p<0.001$ ), in line with what was observed in the other groups (Figure 2C).

When contrasting the performance of the SC group with the one of the CB group, no significant differences could be detected across all durations (all $p>0.29$, corrected). Results clearly revealed that the processing of fine-grained temporal details is resilient to the absence of visual input since birth. Conversely, the LB group performance was impaired for almost all of the tested durations as compared to both SC group (40, 91, 209, 478, 2500ms; all $p<0.03$, corrected) and CB group (40, 91, 209, 478ms; all

$211 \mathrm{p}<0.001$, corrected).

212 Results suggested an altered capability of late blind participants to discriminate sounds when the most 213 efficient strategy is to base performance on local features. These findings reveal that visual input in early 214 phases of development is not a prerequisite to acquiring the ability to discriminate sounds according to their local features. However, this process is encumbered by late-onset blindness (Figure 2D).

\section{Visual deprivation does not impact statistical averaging}

218 By comparing the performances in Texture Discrimination among the three groups, it was possible to address the impact of visual experience on statistical averaging efficiency.

220 The performance of the SC group for any of the selected duration (40,91, 209, 478, 1093, 2500ms) did not differ from both the CB group (all $p>0.47$, corrected) and the LB group (all $p>0.06$, corrected). When comparing the accuracy of the CB group with the one of the LB group, LB performed better for trials where stimuli were $40 \mathrm{~ms}$ long (adjusted $p=0.01$ ). No other difference was observed for all the remaining durations $(91,209,478,1093,2500 \mathrm{~ms}$; all $p>0.09$, corrected; Figure 2E). Altogether, these results reveal that visual deprivation does not significantly influence the process by which auditory statistics are computed over time and used to identify different sound sources.

Relative difference between experiments reveals no advantages for LB when local features should support the performance

For each participant, the accuracy scores in Texture Discrimination were subtracted from the ones in significantly smaller for very short durations in LB group (LC vs. SC: 40, 91; all $p<0.02$, corrected; LC vs. CB: $40 p<0.001$, corrected, $91 p=0.04$, uncorrected) and larger for longer ones (LC vs. SC: 209, 478, 2500; all $p<0.03$, corrected; LC vs. CB: $478 p<0.03$, corrected; see Figure 2F). Conversely, the relative difference of performance in the two Experiments between $\mathrm{CB}$ and SC did not differ for any of the durations (all $p>0.32$, corrected; Figure $2 F$ ) 
238 These results showed that both $\mathrm{CB}$ and SC performed according to predictions showing specific advantages in one experiment or the other according to stimulus duration. On the contrary, the LB group did not display a boost for those conditions in Exemplar Discrimination in which local feature processing

A

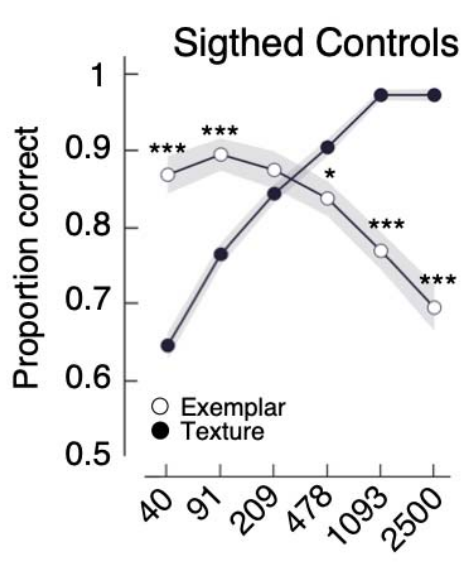

D

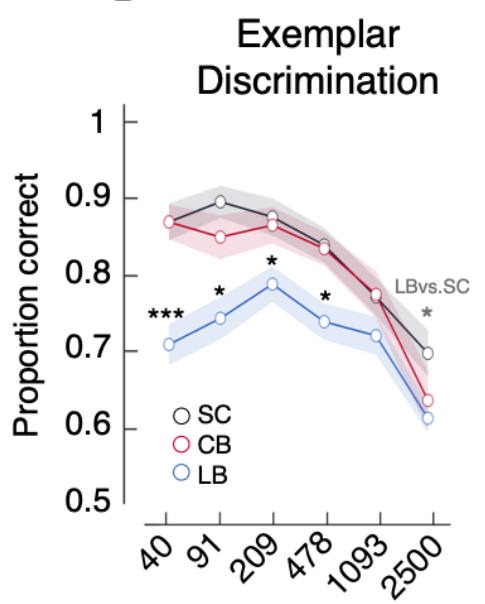

B

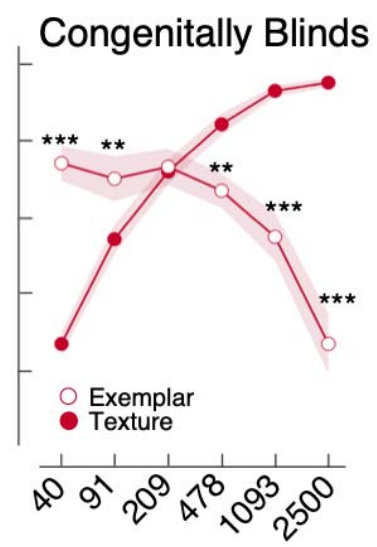

$\mathbf{E}$

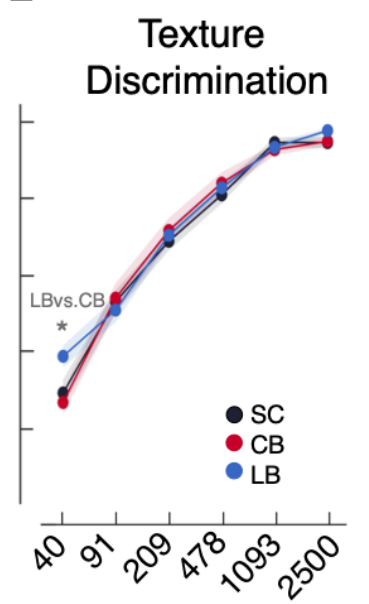

C

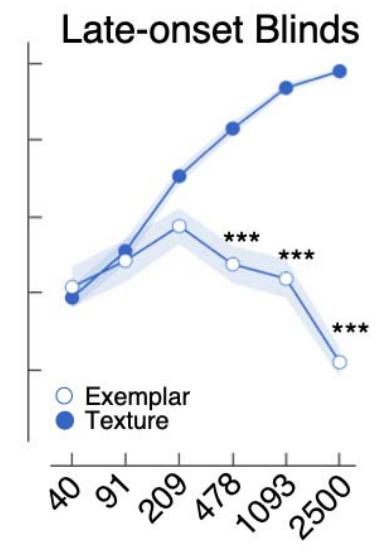

$\mathbf{F}$

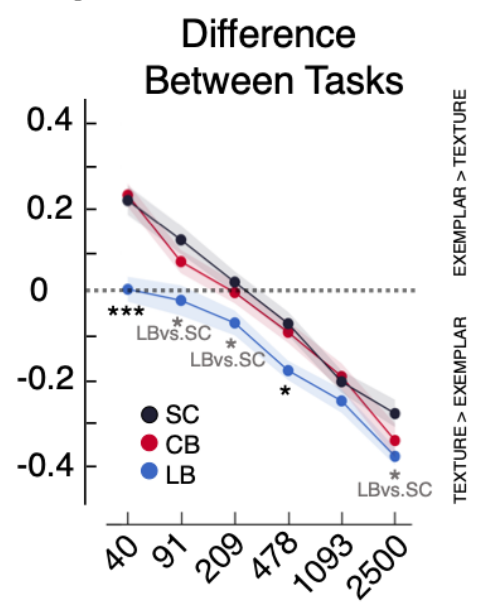

Excerpt duration (ms)

Figure 2. Proportion of correct answers in the two Experiments.

$(A, B, C)$ Results for Exemplar Discrimination vs. Texture Discrimination in each group. Proportion of correct answers across individuals at the group level are shown as a function of excerpt duration.

(D) Between Group comparisons in Exemplar Discrimination. SC and CB groups' performance did not differ. The LB group showed an impaired performance compared to the other groups: late sight loss has a detrimental impact when performance could benefit from higher local features variability.

(E) Between Group comparisons in Texture Discrimination. No significant differences were observed between SC and CB groups. LB performed better for 40ms stimuli compared to CB. 
(F) Relative difference between experiments for all three groups. For each group and duration, the averages across participants of the differences between the performance in the two tasks are displayed. Positive values are found when performance in Exemplar is better than in Texture Discrimination, while negative values represent better performance in Texture than Exemplar Discrimination. Unlike the other two groups, LB never showed an advantage in Exemplar Discrimination compared to Texture Discrimination.

Shaded regions show interpolated standard error of the mean (SE) at each point. Results were corrected for multiple comparisons using the false discovery rate (FDR). If more than one comparison is significant, stars refer to the lower bound; gray stars indicate that only the labeled comparisons were significant; ${ }^{* * *}$ $\mathrm{p}<.001 ;{ }^{* *} \mathrm{p}<.01 ;{ }^{*} \mathrm{p}<.05$.

(See also Figure S4).

\section{Ruling out possible confounds}

243 It could be argued that results in the LB group were idiosyncratic of the participants included in the study.

244 However, the sample size was adequate ( $N=18$ per group) and relatively large compared to previous

245 investigations on blind individuals. All groups were matched adequately by several main variables (size, 246 age, and gender); all participants had no documented auditory deficits, cognitive impairments, or 247 neurological disorders (blindness was associated only to peripheral damage; Table 1). Moreover, LB 248 participants' performance was hampered only in one of the two experiments. In contrast, the observed 249 results in Texture Discrimination were indistinguishable from the ones of the remaining groups for most of 250 the selected durations. If anything, LB group performance was significantly more efficient at $40 \mathrm{~ms}$ as 251 compared to $C B$ and partially $S C$ ( $p<0.04$, uncorrected). In light of these observations, it was possible to 252 exclude that a general cognitive or acoustic impairment could explain the lack of advantage specific for 253 Exemplar Discrimination.

254 To rule out other possible confounds, we ran specific tests on our sample data. Providing the temporal 255 presentation of triplets in the employed 2AFC protocol, it could have been possible that a particular 256 disposition bias towards the first or last interval was present and diverged among groups, at least partially 257 accounting for different results. This was not the case, as all groups did not significantly differ in terms of 258 predisposition towards stimulus intervals (Figure S2A).

259 Another possible confounding effect could have been a between-group difference associated with 260 learning or tiredness within an experimental session. It could have been possible that LB group 261 performance had changed as the experiment proceeded (e.g., progressively diminishing across runs, 262 specifically in the Exemplar Discrimination). However, this was not the case. Comparing accuracy across 263 runs, a similar trend in all groups emerged for each duration (Figure 2D). 
bioRxiv preprint doi: https://doi.org/10.1101/2020.12.16.423049; this version posted June 1, 2021. The copyright holder for this preprint (which was not certified by peer review) is the author/funder, who has granted bioRxiv a license to display the preprint in perpetuity. It is made available under aCC-BY-NC-ND 4.0 International license. 
272 The present study addressed whether visual experience at different phases of the lifespan exerts a cross-

273 modal influence over specific basic auditory computations. No difference was observed between CB and

274 SC groups. Conversely, we found that LB's performance was selectively impaired compared to both SC 275 and $C B$, for those conditions in which optimal performance relied on local features. These results 276 provided evidence that visual experience is not a prerequisite for the full emergence of auditory statistics.

277 Sight loss can negatively impact specific computations only when vision was available in early 278 development and went missing afterward. This outcome is in line with a model in which, in individuals with 279 typical development, visual interactions support auditory segregation in challenging instances accounting 280 for reorganizations and detrimental effects only when visual input goes missing.

\section{Auditory statistics processing develops regardless of visual input availability}

283 Blindness represents a natural state in which it is possible to address cross-modal interdependencies of sensory functions. By comparing CB with SC groups, our study investigated on one hand whether visual input was necessary for the typical development of the two auditory modes of representation underlying the auditory statistics processing, and on the other hand, whether compensatory mechanisms emerged due to lack of vision since birth. Therefore, two different outcomes could have been expected. First, if visual experiences were necessary to properly develop the ability to process local features and/or to compute average statistics, we could have observed an impaired performance of CB participants in one of the two experiments, or both, compared to sighted individuals. For example, evidence exists that CB individuals fail at performing auditory space bisection tasks (Gori et al., 2014), and this observation provides evidence that visual experience is necessary to efficiently encode Euclidian coordinates of sounds (Gori et al., 2014; Gori, Amadeo, and Campus, 2020a,b). Our results provide strong evidence for the relative independence of the development of auditory statistic processing from early visual experience.

On the other hand, coherently with previous evidence of enhanced auditory skills in congenitally and early blinds (e.g., Doucet et al., 2005; Muchnik et al., 1991; Röder et al., 1999), lack of vision since birth could have improved the sensitivity to the auditory information included in sound textures, leading to the emergence of specific compensatory mechanisms. For instance, scattered evidence suggests that blind individuals are able to understand syllables at a greater time speed compared to natural speech (Moos and Trouvain, 2007; Trouvain, 2007; Dietrich, Hertrich and Ackermann, 2013), an ability perhaps supported by a higher auditory sampling rate capacity. We could have expected the CB group to be able to retain more local features in time before averaging statistics, showing a better performance for longer durations in Exemplar Discrimination. A compensatory mechanism favoring the statistical averaging mode would have led to better results in Texture Discrimination. Furthermore, a better frequency tuning (Huber et al., 2019) to temporal local features in CB would have enhanced the sensitivity to small acoustic 
differences, leading to superior-to-sighted performances for short excerpts both in Exemplar and Texture Discrimination. However, none of these hypotheses was confirmed in our data, as the performance was indistinguishable between $\mathrm{CB}$ and SC groups. The absence of compensatory mechanisms -arising due to the lack of vision since birth- suggests these basic auditory computations cannot be improved by crossmodal adaptations associated to early blindness.

312 Previous evidence already suggested the existence of auditory functions which develop independently 313 from visual experience since birth (for instance, auditory gap detection threshold; Weaver and Stevens, 314 2006; pure tone threshold audiometry and acoustic reflex threshold; Starlinger and Niemeyer, 1981). 315 Similarly, it seems that the development of auditory computations tested in this study is not influenced by 316 the availability of other senses.

317 We can argue that the extraction of the auditory statistics necessary for textures recognition occurs at a 318 very basic level of processing, while functions that showed visual input dependencies are mostly the 319 result of higher-order cortical operations. Evidence suggests that multisensory functions performed at 320 subcortical levels rely less on experience compared to cortical multisensory functions (e.g., Putzar et al., 321 2010). As the auditory model used in our study to extract and impose statistics represented peripheral 322 computations, from the cochlea up to the midbrain (McDermott and Simoncelli, 2011), it is possible that 323 the functional development of the set of auditory statistics included in our stimuli is exclusive competence 324 of the auditory system and does not rely on other modalities, such as vision. Further studies including 325 statistics resulting from computations beyond primary cortex (Chi, Ru and Shamma, 2005; Norman326 Haignere and McDermott, 2018) and involving other types of naturalistic stimuli will be able to shed light on potential visual influences over the development of higher-order auditory statistics.

\section{Functional interplay between selective auditory computations and vision}

330 By comparing CB, SC, and LB groups, we can affirm that while early vision is not necessary for the typical development of the auditory statistics processing, the lack of vision can still influence the processing of

332 local features. No compensatory effects were found in LB group for any of the tested computational modes, while a detrimental effect was present for the acoustic local features analysis.

334 One possibility for this mode to be affected in LB, but not CB, individuals is that a visual influence on 335 auditory computations can only take place after the major development of the auditory system has 336 occurred. Consistent evidence shows that, even considering basic auditory functions, human 337 performance keeps improving gradually for over nearly a decade. Frequency discrimination, such as 338 perceiving differences between two tones presented sequentially, does not mature until roughly 10 years 339 of age for low frequencies (Maxon and Hochberg, 1982; Jensen and Neff, 1993; Moore et al., 2011). 340 Similarly, the thresholds for detecting amplitude modulations become adult-like only after 10 years of age 341 (Banai, Sabin and Wright, 2011). The use of local features comprised a number of operations, including 342 the measurement of amplitude modulations over time (Dau, Kollmeier and Kohlrausch, 1997). Thus, it is 
343 possible they are designed to develop independently from visual input and only after their functional 344 development is completed, interactions across senses are allowed.

345 The observed effects might also depend on the development of multisensory functions. Previous studies

346 have revealed that certain aspects of multisensory integration can occur only by the age of 8-10 years

347 (Gori et al., 2008). In the same vein, basic audio-visual (AV) multisensory facilitations have been found

348 immature until the age of 9 years old while similar patterns have been observed between adolescents and 349 adults (Brandwein et al., 2011). Similarly, the development of multisensory speech perception has been 350 found to progress until late childhood (Ross et al., 2011). The present data might suggest that the 351 interactions between basic auditory computations tested here and vision occur after full development of 352 AV multisensory functions. The availability of visual input in early developmental phases would prompt 353 the typical development of AV interactions. As a result, the sudden and permanent loss of visual input 354 could, in turn, alter auditory processing which had typically developed.

355 Shamma (2001) suggested that the most essential auditory percepts (timbre, pitch and localization) can 356 be derived through computational approaches typically employed to model visual processing (see also 357 McDermott and Simoncelli., 2011). As an example, extracting the profile of sound spectrum has been 358 associated to the type of neural computations which are necessary for the extraction of the form of an 359 image. The common principle could rely on lateral inhibition for the enhancement of edges or peaks (Lyon 360 and Shamma, 1996; Shamma, 1985). The present results might suggest that if a unified plan for basic 361 auditory and visual computations exists, it might develop, for certain functions, independently for each 362 sensory modality.

363 The absence of associations between the accuracy in both tasks and duration or onset of blindness 364 (Figure S5) suggested that the performance: (i) was not influenced by the number of years people were 365 visually deprived (in our LB sample, duration of blindness spanned from 2 years up to 28 years); (ii) was 366 similar for people who became blind during childhood (10 years of age) as well as during adulthood (up to 36751 years of age, in our LB sample). However, we had the chance to test two early blind individuals whose 368 blindness onset occurred during their third year of life (Figure S3). Remarkably, their results 369 systematically overlapped with CB and SC groups, providing further support to the evidence that the 370 dampening of local features processing manifests only if sight loss occurs relatively late in the 371 development.

372 Finally, the deficit observed in the LB group does not exclude that limiting local features accessibility can 373 provide ecological advantages. Identifying a Sound Texture equals recognizing the statistics included in 374 its sound waveform (McDermott and Simoncelli, 2011). Thus, it is possible that relying on statical 375 averaging only when information is consistent -at the expense of temporal details- prompts sound-object 376 recognition in an everyday environment. The results in our LB group could suggest a form of adaptive 377 perceptual learning (Watanabe and Sasaki, 2015) relying on implementing strategical changes aimed at 378 facing a remarkable loss in the overall available sensory input (de Villers-Sidani and Merzenich, 2011). 
bioRxiv preprint doi: https://doi.org/10.1101/2020.12.16.423049; this version posted June 1, 2021. The copyright holder for this preprint (which was not certified by peer review) is the author/funder, who has granted bioRxiv a license to display the preprint in perpetuity. It is made available under aCC-BY-NC-ND 4.0 International license. 
CONCLUSION

385

386 Overall, this evidence has several important implications. First, it discloses how basic auditory

387 computations can develop independently from early visual input. Second, it shows a selective

388 detrimental effect induced by a late-onset sight loss over selective, non-spatial aspects of the 389 auditory processing. Third, it has the potential to expand our approaches in several fields,

390 including auditory, visual, multisensory, and brain disorders research. Moreover, it provides novel 391 ground for applied science such as sensory substitution device and auditory rehabilitation

392 strategies for people who lost vision compared to those born blind. Finally, we proved that 393 combining computational methods with human models of sensory deprivation provides the 394 context to assess the degree of plasticity of specific computations performed by the sensory 395 systems. While the present study focused on the auditory domain, similar approaches could be 396 employed for other modalities. Providing the resemblance of the Auditory Texture Model 397 (McDermott and Simoncelli, 2011) with a previously validated Visual Texture Model (Portilla and 398 Simoncelli, 2000), and the presence of textures in other modalities, such as touch (Picard et al., 399 2003; Weber et al., 2013), it can be possible to address with similar approaches the development 400 and sensory interplays across computations performed by other senses.

401

402

403

404

405

406

407

408

409 


\section{MATERIALS AND METHODS}

423 Experimental procedures and methods are inspired by the work of McDermott, Schemitsch, and

424 Simoncelli (2013).

426 Synthetic texture synthesis

427 Synthetic textures were synthesized from the model described in a previously published paper 428 (McDermott and Simoncelli, 2011). For synthesis, we used the MATLAB-based Sound Texture 429 Synthesis Toolbox, available here: http://mcdermottlab.mit.edu.

430 Auditory statistics were computed from different signals of 7-s length, each one being an Original 431 Recording of a natural Sound Texture, with a sampling rate of $20 \mathrm{kHz}$. These sounds are also 432 available on the website previously cited. The Original Recordings we used for synthesis and 433 experiment were a subset of the ones used by McDermott, Schemitsch, and Simoncelli (2013).

434 For each original recording, cochlear envelopes and modulation bands were extracted from the 435 signal, by filtering it through the Auditory Texture Model (McDermott and Simoncelli, 2011). 436 Precisely, extracted statistics included marginal moments (mean, variance, and skew) of each 437 cochlear envelope, envelope cross-correlation, the normalize power in each modulation band, 438 and two correlations between modulation bands ( $\mathrm{C} 1$ and $\mathrm{C} 2$ ). Kurtosis was omitted from the 439 computation (see toolbox-authors' suggestion). Statistics were measured with a temporal 440 weighting function that faded to zero over the first and last second of the original recordings to 441 avoid boundary artifacts (McDermott and Simoncelli, 2011).

442 After setting the parameters, the process was initialized from a 5-s white noise sample. The noise 443 signal was again filtered through the model and the original sound statistics were imposed on its 444 cochlear envelopes, with circular boundary conditions. The resulting envelopes were multiplied by 445 their associated fine structure and recombined into the signal. The procedure was repeated in an 446 iterative way, until the imposed statistics measured from the synthetic signal reached a desirable 447 signal-to-noise-ratio (SNR) of 30-dB, which represents the ratio of the squared error of a statistics 448 class, summed across all statistics in the class, to the sum of squared statistics values of that 449 class. The average of each statistics class was at least 20-dB (McDermott and Simoncelli, 2011). 450 The resulting Synthetic Sound is a 5-s random signal constraint only by the over-imposed 451 statistics of interest (Figure 1A). The procedure was repeated four times on four different white 452 noise samples to obtain four different synthetic exemplars for each Sound Texture (adapted from 453 McDermott, Schemitsch, and Simoncelli, 2013).

454

455 Stimuli

456 Each synthetic exemplar was cut into excerpts of different durations, equally spaced on a 457 logarithmic scale (40, 91, 209, 478, 1093, and 2500ms). A 10-ms Hanning window was applied to 
458 the beginning and the ending segments of each excerpt, to smooth signal onset and offset. All excerpts were equalized to the same root-mean-squared level $(r m s=0.1)$.

\section{Experimental Procedures}

462 Participants sat in front of a computer and performed the task using the mouse. Experiments 463 were implemented in MATLAB. All subjects were blindfolded by a mask that could filter almost $464100 \%$ of the light and kept their eyes closed. Stimuli were played on a Macbook Pro 2017, with a 465 built-in sound card with a frequency sampling rate of $44.1 \mathrm{~Hz}$, through a headphone set Audio 466 Technica Pro ATH-M50X, at a volume of c.a. $75 \mathrm{~dB}$ SPL, which was kept constant for each 467 stimulus.

468 The task was very similar to the one in the original paper by McDermott, Schemitsch, and 469 Simoncelli (2013) but modified to be suitable for visually deprived individuals. In fact, no visual 470 interaction with the screen was required and audio instructions were provided through the 471 headphones in Italian or English, according to participant's preferred language.

472 Participants performed in two sessions, each comprising either version of the two experiments 473 (Exemplar Discrimination or Texture Discrimination), presented in a counterbalanced order 474 across subjects. Both sessions were performed in the same day with a half-an-hour break in 475 between; each session lasted approximately 35 - 40 minutes. 54 Sound Textures were employed 476 in Texture Discrimination and 36 in Exemplar (see Table S1, Supplementary information); each 477 experiment comprised 216 trials. Every 54 trials, corresponding to about 6-7 minutes of 478 stimulation, participants were allowed to take a few-minutes break, for a total of four runs per 479 each session, separated by four small breaks.

480 In both sessions, each trial consisted of a triplet of sounds of the same duration. Stimuli duration 481 could vary across trials, and six durations were employed (either 40, 91, 209, 478, 1093 or 482 2500ms). The number of trials per duration was equal across all the six possible durations (36 483 trials for each one) and stimuli were presented in a randomized order. To control for a stimulus 484 expectancy effect, inter-stimulus interval (ISI) could vary between 400, 500, 600, 700, and 800485 ms. Participants were asked to be as accurate as possible in their choice and there was no time486 limit to answer. Once the participant had responded, the presentation of the next triplet occurred 487 after a short pause lasting between 2 and 2.5 -s, with steps of $100 \mathrm{~ms}$.

488 Test sessions were performed before both of the actual experimental sessions, to make sure 489 participants understood the tasks and to have them familiarize with the type of stimuli. Test stimuli 490 were drawn randomly across the 36 texture pairs (Table S1, Supplementary information); 491 excerpts used in the trial session were then excluded by the actual experiment. Three trials for 492 each duration were presented in a random order during test sessions (for a total of 18 trials per 493 duration). A feedback was provided only during the trial session and consisted in an audio 
message stating if their response was correct or incorrect. Feedback was not provided during experimental sessions, following the protocol by McDermott, Schemitsch, and Simoncelli, 2013.

\section{Exemplar Discrimination}

498 Two excerpts coming respectively from two exemplars of the same Sound Texture were selected.

499 Both excerpts were extracted at the same time point along the 5-s segments. Excerpts were then

500 presented as triplet of sounds in a trial: consequently, one of the two excerpts was repeated

501 twice, while the third sound was the other. The different sound could be located as the first one or

502 the last one in the triplet, and this varied randomly across trials.

503 Participants were informed that two stimuli will be identical and were asked to indicate which

504 stimulus was different from the other two. If they thought it was the first one, they would click the

505 left button of the mouse, otherwise the right one. The middle sound was used as the reference

506 (Figure 1C).

507

\section{Texture Discrimination}

509 Three excerpts were drawn among three different exemplars coming from pairs of Original Sound

510 Textures: two of the excerpts came from two different exemplars of the same Sound Texture

511 while the third one from an exemplar of another Sound Texture. Sound Textures were paired

512 according to similarity, as done by McDermott, Schemitsch, and Simoncelli, 2013; Table S1,

513 Supplementary information) and the different exemplar was a synthetic sound derived from the

514 same white noise of one of the other two exemplar, so their original associated fine structure was

515 the same, while only the imposed statistics were different. All three excerpts were extracted at the

516 same time point along the 5-s segments. Again, the three excerpts were presented in a trial so

517 that all the sounds were different, but two of them would originate from the same Sound Texture,

518 while the other one had a different sound source. Participants were made aware of the fact that

519 all sounds could be different and were asked to report which one came from the diverging

520 acoustic source. Some examples were provided in order to facilitate task comprehension (i.e.,

521 "Two sounds can be the sound of a fireplace, the other one is the sound of the rain"). The correct

522 answer could be either the first or last sound in the triplet, while middle one had to be used has a

523 reference. Middle excerpt and deviant excerpts were derived from the same white noise sample.

524 If participants thought the deviant was the first sound, they would click the left button of the 525 mouse, otherwise the right one (Figure 1B).

\section{Average statistical variability}

528 Standard deviation of a set of employed envelope statistics (envelope mean, variance, skew, 529 cross-correlation, and modulation power) was measured between couples of excerpts presented 530 in both experiments. The pair could be made of excerpts coming from different exemplar of the 
531 same Sound Texture (column 1, Table S1, Supplementary information) or excerpts coming from

532 different Sound Textures (column 1 and 2, Table S1, Supplementary information; Figure 1D). The

533 average of all statistics variability among couples of sounds was computed to make predictions

534 about performance. When both excerpts originated from different exemplars of the same Sound

535 Textures, their variability tended toward zero as duration increased, whereas opposite trend was

536 observed when stimuli came from different Sound Textures. Moreover, differences in variability

537 between the two conditions (Different Exemplar vs. Different Texture) increased with duration

538 (Figure 1D). Separately for each of the aforementioned statistics, we performed pairwise

539 comparisons (t-tests, FDR corrected) between the two conditions (Different Exemplar vs.

540 Different Texture) and observed that variability between couples of excerpts coming from

541 different Sound Textures, as compared to ones originating from different exemplar of the same

542 Texture in envelope mean was larger for all durations (all $p>0.05$, corrected); in envelope

543 skewness in was larger at duration 91, 478, 1093, 2500ms (all $p>0.05$, corrected); variability

544 significantly diverged only at long durations in envelope variance, envelope cross-correlation

545 (478ms, 1093, and 2500ms; all $p<0.001$, corrected), and modulation power (1093, 2500ms; all

$546 \mathrm{p}<0.01$, corrected; Figure S1). Analyses were performed using MATLAB.

547

548 Participants

549 Data from three groups of participants, matched for size, gender, and age, were analyzed in this 550 study. We recruited a group of congenitally blind individuals $(C B ; N=18 ; F=9$; mean age = 55137.06 years; std $=10.75$ ). Data of the $18 \mathrm{CB}$ individuals were used as reference for the 552 recruitment of a group of late blind individuals and a group of sighted controls. Each CB individual 553 in our sample was matched with a sighted individual and a LB individual of same gender and 554 similar age (mean age of groups was within 2 std of difference), for a total of 18 participants for 555 each group. Late onset blind individuals ( $\mathrm{LB} ; \mathrm{N}=18 ; \mathrm{F}=9$; mean age $=40.11$; $\mathrm{std}=12.68$ ); range 556 of blindness onset $=10-51$ years; range of blindness duration= 2-28 years), and sighted controls 557 (SC; $N=18 ; \mathrm{F}=9$; mean age $=38.06$ years; $\mathrm{std}=12.93$ ).

558 Before experimental session began, all blind participants underwent a short interview to gather 559 several information, especially about onset, cause, and duration of blindness, together with other 560 anamnestic information.

561 All participants in the final sample were healthy and fully understood the task requests. During 562 recruitment, exclusion criteria comprised documented hearing impairment (i.e., acoustic implants, 563 tinnitus), neurological disorders. The data of 3 CB individuals, 1 early blind participant (blindness 564 onset: 7 months) and of 2 SC were not included in the final sample and thus were not analyzed 565 due to their inability to perform/terminate one or both sessions or being easily distracted during 566 experimental sessions (i.e., participant often asked questions and talked during the task). For all 
567 of the blind participants in the sample, blindness was total and caused only by peripherical 568 pathologies (Table 1).

569 Two early blind (EB) participants were also tested (EB1, gender $=M$; age $=26$, blindness onset $=$

570 3years; $E B 2$, gender $=M$; age $=24$, blindness onset $=3$ years). Results from these participants

571 were excluded from the analyses, as blindness onset was borderline between blind groups. Their

572 data are plotted in Supplementary information (Figure S3).

573 Beforehand, all participants were informed about the procedures and purpose of the study and

574 signed a written informed consent prior to testing. The study was approved by the regional Ethical

575 Committee (CEAVNO protocol $n$ 24579). The study protocol adhered to the guidelines of the

576 Declaration of Helsinki (2013).

577

\section{Statistical Analyses}

579 Proportion of correct responses for each individual was used as dependent measure for statistical

580 analyses. To assess that sample data were normally distributed, we performed both Kolmogorov-

581 Smirnov and Shapiro-Wilk tests with the average of proportion of correct answers in each

582 experiment (separately) as dependent variables. In each test, dependent variable was split

583 according to group. Both tests indicated that data were normally distributed in both experiments

584 and for all of the groups, giving significance values higher than 0.05 (see Supplementary

585 information, Table S2).

586 We performed an ANCOVA using IBM SPSS Statistics for Macintosh, Version 26.0. The model

587 included the between-participants factor Group (SC, CB, LB) and two within-participant factors:

588 Experiment (Exemplar Discrimination vs. Texture Discrimination) and Duration (40, 91, 209, 478,

589 1093, 2500); given the relatively large age-range across the entire sample (20-62 years old), age

590 was included as a covariate. Since groups were matched, age unlikely accounted for between-

591 groups effects, but it could still have had an impact at the individual level.

592 Mauchly's test indicated that the assumptions of sphericity had been violated for the interaction

593 Experiment * Duration $(\mathrm{c} 2(2)=25.62, \mathrm{p}<0.05)$. Thus, degrees of freedom were corrected using

594 Greenhouse-Geisser estimates of sphericity $(\varepsilon=0.81)$.

595 There was a significant main effects of Duration, $F_{(5,250)}=9.45, p<0.001, \eta^{2}=0.16$, and its

596 interaction with the factor Experiment, $F_{(4.07,203.35)}=15.91, p<0.001, \eta^{2}=0.24$. Also, there was a

597 significant main effect of the between-participants factor Group, $F_{(2,50)}=4.99, p=0.01, \eta^{2}=0.17$,

598 and a significant interaction between factors Group and Experiment, $F_{(2,50)}=8.35, p=0.001$, with

599 a large effect size of $\eta^{2}=0.25$. Moreover, there was a significant interaction effect among

600 Experiment, Duration, and Group, $F_{(10,250)}=3.29, p=0.001$, with a medium-to-large eta-squared

601 of $\eta^{2}=0.12$. Participant's age and its interactions with all other independent variables in the

602 model were non-significant (all $p>0.14$ ). 
603 To break down the three-way interaction, we performed planned pairwise comparisons, using 604 two-sided t-tests only on pre-specified effects of interest, as opposed to investigating all main 605 effects and interactions as in exploratory analyses (see Cramer et al., 2016).

606 The pre-specified contrasts were: (i) Within Group contrasts, for each group (CB, LB, SC) and 607 duration (40, 91, 209, 478, 1093, 2500): Exemplar Discrimination vs. Texture Discrimination), for 608 a total of 18 contrasts (Figure 2A, 2B, 2C). (ii) Between Group contrasts (within durations and 609 experiments). For each duration $(40,91,209,478,1093,2500)$ and experiment (Exemplar 610 Discrimination vs. Texture Discrimination): LB vs. SC, CB vs. LB, LB vs. CB, for a total of 36 611 contrasts (Figure 2D, 2E). (iii) Within Experiment contrasts (within Group and across duration). 612 For each experiment (Exemplar Discrimination and Texture Discrimination) and group (CB, LB, 613 SC): 40 vs. either $91,209,478,1093$ or 2500; 91 vs. either 209, 478,1093 or 2500; 209 vs. either 614478,1093 or 2500 ; 478 vs. either 1093 or $2500 ; 1093$ vs. 2500 . For a total of 90 contrasts. P615 values were corrected for multiple comparisons across all 144 pre-specified pairwise 616 comparisons (the contrasts of interest listed above) using the false discovery rate (FDR; 617 Benjamini, Drai, Elmer, Kafkafi, and Golani, 2001) and a q-value of 0.05. Post-hoc comparisons 618 and adjusted $p$-values were computed using RStudio version 1.2.1335.

\section{Within Group contrasts}

\section{Sighted controls}

622 For the SC group, the within Group contrasts Exemplar Discrimination vs. Texture Discrimination 623 were significantly different at durations 40 ( $p<0.001$, corrected), 91 ( $p<0.01$, corrected), 478 ( $p$ $624<0.05$, corrected), 1093 ( $p<0.001$, corrected), and 2500 ( $p<0.001$, corrected). For short 625 durations $(40,91)$, performance was higher in Exemplar Discrimination (mean proportion correct: $62640 \mathrm{~ms}=0.87 ; \mathrm{SE}=0.02 ; 91 \mathrm{~ms}=0.89 ; \mathrm{SE}=0.02$ ) compared to Texture Discrimination (mean 627 proportion correct: $40 \mathrm{~ms}=0.65 ; \mathrm{SE}=0.02 ; 91 \mathrm{~ms}=0.77 ; \mathrm{SE}=0.02$ ). Conversely, for longer 628 durations $(478,1093,2500)$, performance was better for Texture Discrimination (mean proportion 629 correct: $478 \mathrm{~ms}=0.91 ; \mathrm{SE}=0.01 ; 1093 \mathrm{~ms}, 0.97 ; \mathrm{SE}=0.01 ; 2500 \mathrm{~ms}=0.97 ; \mathrm{SE}=0.01$ ) 630 compared to Exemplar Discrimination (mean proportion correct: $478 \mathrm{~ms}=0.84$; $\mathrm{SE}=0.02$; $6311093 \mathrm{~ms}, 0.77 ; \mathrm{SE}=0.03 ; 2500 \mathrm{~ms}=0.70 ; \mathrm{SE}=0.03$ ). No Difference was observed at duration 632209 (mean proportion correct at 209: Exemplar $=0.88 ; \mathrm{SE}=0.02 ;$ Texture $=0.85 ; \mathrm{SE}=0.01 ; \mathrm{p}>$ 633 0.05, corrected; Figure 2A).

\section{Congenitally blind group}

636 For the CB group, results pattern was remarkably similar to SC's. The contrasts Exemplar 637 Discrimination vs. Texture Discrimination were significant at duration $=40(p<0.001$, corrected), 63891 ( $p<0.01$, corrected), 478 ( $p<0.001$, corrected), 1093 ( $p<0.001$, corrected), and 2500 ( $p<$ 6390.001 , corrected), with performance being higher for short durations in Exemplar Discrimination 
640 (mean proportion correct: $40 \mathrm{~ms}=0.87 ; \mathrm{SE}=0.02 ; 91 \mathrm{~ms}=0.85 ; \mathrm{SE}=0.03$ ) compared to Texture 641 Discrimination (mean proportion correct: $40 \mathrm{~ms}=0.65$; $\mathrm{SE}=0.02 ; 91 \mathrm{~ms}=0.77 ; \mathrm{SE}=0.02$ ). On 642 the contrary, for long durations, performance was higher in Texture Discrimination (mean 643 proportion correct: $478 \mathrm{~ms}=0.92$; $\mathrm{SE}=0.01 ; 1093 \mathrm{~ms}, 0.97 ; \mathrm{SE}=0.01 ; 2500 \mathrm{~ms}=0.98 ; \mathrm{SE}=$ 6440.01 ) compared to Exemplar Discrimination (mean proportion correct: $478 \mathrm{~ms}=0.83$; $\mathrm{SE}=0.02$; $6451093 \mathrm{~ms}=0.77 ; \mathrm{SE}=0.03 ; 2500 \mathrm{~ms}=0.64 ; \mathrm{SE}=0.04)$. As for the SC group, no Difference was 646 observed at duration $=209$ (mean proportion correct at 209: Exemplar Discrimination = 0.87; SE $647=0.02$; Texture Discrimination $=0.86 ; \mathrm{SE}=0.02 ; \mathrm{p}>0.05$, corrected; Figure $2 \mathrm{~B}$ ).

\section{Late-onset blind group}

650 For the LB group, comparisons between Exemplar Discrimination vs. Texture Discrimination 651 revealed a substantially different pattern of results compared to CB and SC groups. The 652 performance of the two tasks did not differ at short durations 40 (mean proportion correct: 653 Exemplar Discrimination $=0.70$; $\mathrm{SE}=0.03$; Texture Discrimination $=0.69$; $\mathrm{SE}=0.01$ ), 91 (mean 654 proportion correct: Exemplar Discrimination $=0.76$; $\mathrm{SE}=0.03$; Texture Discrimination $=0.74$; SE $655=0.02$ ), 209 (mean proportion correct: Exemplar Discrimination $=0.85$; SE $=0.02$; Texture 656 Discrimination $=0.79 ; \mathrm{SE}=0.01$; all $\mathrm{p}>0.07$, corrected). Significant differences emerged only at 657 longer durations 478 ( $p<0.001$, corrected), 1093 ( $p<0.001$, corrected), and 2500 ( $p<0.001$, 658 corrected), where performance was better for Texture Discrimination (mean proportion correct: $659478 \mathrm{~ms}=0.91 ; \mathrm{SE}=0.01 ; 1093 \mathrm{~ms}=0.97 ; \mathrm{SE}=0.04 ; 2500 \mathrm{~ms}=0.99 ; \mathrm{SE}=0.02$ ) compared to 660 Exemplar Discrimination (mean proportion correct: $478 \mathrm{~ms}=0.73$; $\mathrm{SE}=0.01 ; 1093 \mathrm{~ms}=0.72$; SE $661=0.03 ; 2500 \mathrm{~ms}=0.61 ; \mathrm{SE}=0.02 ;$ Figure $2 \mathrm{C}$ ).

662

\section{Between Group contrasts}

664 Contrasting SC vs. CB, no significant difference in the proportion of correct answers was 665 observed in either Experiments (Exemplar Discrimination and Texture Discrimination) and for any 666 of the durations (all $p>0.37$, corrected).

667 In the Exemplar Discrimination, LB vs. SC and LB vs. CB contrasts were significantly different at 668 specific durations: comparisons between LB and SC groups were significant at duration $=40 \mathrm{p}$ $669<0.001$, corrected), 91 ( $p<0.001$, corrected), 209 ( $p<0.05$, corrected), 478 ( $p<0.05$, 670 corrected), and 2500 ( $p<0.05$, corrected); comparisons between LB vs. CB groups were 671 significantly different at duration 40 ( $p<0.001$, corrected), 91 ( $p<0.05$, corrected), 209 ( $p<$ 6720.05 , corrected), and 478 ( $p<0.05$, corrected; Figure 2D).

673 In the Texture Discrimination experiment, LB vs. SC contrasts were not significantly different (all $p$ $674>0.07$, corrected). A significant contrast was observed at duration 40 when comparing LB vs. CB 675 ( $p<0.05$, corrected) with LB performing better than CB. None of the other contrasts at the 676 remaining durations was significant (all $p>0.05$, corrected; Figure $2 E$ ). 


\section{Within Experiment contrasts}

679 Overall, in the Exemplar Discrimination, performance tended to decrease with duration as in 680 McDermott, Schemitsch, and Simoncelli (2013). for both SC and CB groups, but not for the LB 681 group. For the SC group, the following comparisons between durations were significantly 682 different: 1093 vs. 40,1093 vs. 91, 1093 vs. 209, 1093 vs. 478 and 2500 vs. 40,2500 vs. 91, 6832500 vs. 209,2500 vs. 478 (all $p<0.05$, corrected) whereas the other comparisons 40 vs 91,40 684 vs 209,40 vs. 478,91 vs. 209,91 vs. 478,209 vs. 478 were not significant (all $p>0.05$, 685 corrected). Similarly, for the CB group, the following comparisons resulted significant: 40 vs. 6861093,40 vs. 2500,91 vs. 2500,209 vs. 1093,209 vs. $2500 \mathrm{~ms}, 478$ vs. 2500 , and 1093ms vs. $6872500 \mathrm{~ms}$ (all $p<0.05$, corrected). For the LB group, comparisons between durations were all non688 significant (all $p>0.91$, corrected), apart from the comparisons between duration 2500 and all of 689 the others ( 40 vs. 2500,91 vs. 2500,209 vs. 2500,478 vs. 2500,1093 vs. 2500 ) which were 690 significantly different (all $p<0.01$, corrected).

691 The data for Texture Discrimination replicated the one from McDermott, Schemitsch, and 692 Simoncelli (2013) for all groups, with performance progressively increasing with duration.

693 For all the three groups, the following comparisons across durations were significantly different: 69440 vs. either 91, 209, 478,1093, or 2500; 91 vs. either 209, 478,1093 or 2500; 209 vs. either 695478,1093 or 2500; 478 vs. either 1093 or 2500 (SC: all $p<0.01$, corrected; CB: all $p<0.02$, 696 corrected; LB: all $p<0.02$, corrected), while the comparison between the two longest durations, 6971093 vs. 2500 , was significant only for the LB group and not for SC and CB groups (LB: $p=$ 698 0.007, corrected; SC: $p=1$, corrected; CB: $p=0.36$, corrected).

\section{Difference in performance between Exemplar and Texture Discrimination}

701 For every duration, each participant's accuracy-score in Texture discrimination was subtracted 702 from the scores in Exemplar Discrimination (Figure 2F). To test whether there was a significant 703 difference among groups, we ran an ANOVA for repeated measures with one within-subjects 704 factor Duration and one between-subjects factor Group. The dependent variable was the relative 705 difference between the accuracy scores in Exemplar Discrimination and Texture Discrimination.

706 Main effects of Group, $F_{(2,51)}=8.72, p<0.001, \eta^{2}=0.26$, and Duration, $F_{(5,255)}=230.42, p<$ $7070.001, \eta^{2}=0.82$, were significant, together with their interaction, $F_{(10,255)}=3.22, p<0.001, \eta^{2}=$ 708 0.11. We ran FDR corrected pairwise comparisons (two-tailed t-tests; q-value $=0.05$ ) on pre709 selected contrasts of interest highlighting the differences between groups within each duration. All 710 of the comparisons between $\mathrm{CB}$ and SC were not significant (all $\mathrm{p}>0.32$, corrected). 711 Comparisons between LB and CB were significant at duration 40 ( $p<0.001$, corrected), 478 ( $p<$ 7120.03 corrected) and not significant for other durations (all $p>0.09$, corrected). Finally, 713 comparisons between SC and LB were significant for most of the durations (40, 91, 209, 478, 
714 2500; all $p<0.03$, corrected) but 1093 ( $p=0.32$, corrected) (Figure 2D). Absolute values of the 715 average across participants of the differences at the Group level are displayed separately for 716 each group in Supplementary information, Figure S4(A, B, C). For both SC (Figure S4A) and CB 717 (Figure S4B) we observed a U-shaped trend, consisting of higher values at short and long 718 durations and almost zero at intermediate one. On the other hand, in LB groups (Figure S4C) a 719 different trend was observed, with values being almost zero at short durations $(40,91)$ and 720 progressively increasing at longer ones.

\section{Testing for Disposition bias}

723 As participants were asked to choose between first and third intervals, the temporal connotation 724 of this 2AFC protocol could have led to a response disposition bias (e.g., listeners could have 725 shown a tendency toward reporting one of the two intervals, for example the last one). In order to 726 rule out that systematic trends for bias could differ across groups and, to some extent, could 727 account for the impaired performance in LB, for each participant we calculated how many times 728 they overestimated stimuli in one position, as compared to the other. Separately for each 729 experiment and for each duration, the total number of times participants pressed the mouse's left 730 button, stating that deviant sound was the first interval, was subtracted from the total number of 731 times that correct answer was actually the left one. Positive numbers would indicate an 732 overestimation of stimuli in the last interval, whereas negative values would refer to an 733 overestimation of first intervals. In order to check for significant differences, we ran a Repeated734 Measure ANOVA with total number of overestimated stimuli in one position as dependent 735 variable, Group as between-subjects factor, and Experiment and Duration as within-subjects 736 factors. As Mauchly's test indicated that the assumptions of sphericity had been violated for the 737 effect Duration $(c 2(2)=76.28, p<0.001)$, the interactions Experiment ${ }^{\star}$ Duration $(c 2(2)=78.76, p$ $738<0.001$ ), degrees of freedom were corrected using Greenhouse-Geisser estimates of sphericity 739 (Duration, $\varepsilon=0.58$; Experiment ${ }^{\star}$ Duration, $\varepsilon=0.56$ ). No significant effects were found for any 740 within-subjects factors, nor for the between-subject factor Group and their interactions (all $F$ > 741 0.07). Statistics were carried out using IBM SPSS Statistics for Macintosh, Version 26.0. Data are 742 displayed in Figure S2A.

\section{Testing for Learning Effect}

745 Sessions were divided into four runs of 54 trials each. In order to check for the occurrence of 746 divergent learning and/or tiredness effects between groups, within each experiment and duration, 747 total number of correct answers in run 1 was used as a baseline and was subtracted from the 748 number of correct answers in the next runs (run 2, 3, and 4). Positive values meant that 749 performance increased compared to the first run, showing a learning effect, whereas negative 750 values were associated with a decrease in the performance, possibly a tiredness effect. We ran a 
751 Repeated-Measure ANOVA using IBM SPSS Statistics for Macintosh, Version 26.0, with

752 baseline-subtracted correct answers as the dependent variable, Group as between-subjects

753 factor, and Experiment, Duration, and Run within-subjects factors. Mauchly's test indicated that

754 the assumptions of sphericity had been violated for the main effect of Duration (c2(2) $=67.35, p<$

755 0.001), the interactions Experiment ${ }^{\star}$ Duration $(\mathrm{c} 2(2)=38.80, \mathrm{p}<0.001)$, Duration* Run $(\mathrm{c} 2(2)=$

$756150.31, p<0.001)$, and Experiment ${ }^{\star}$ Duration*Run $(c 2(2)=212.37, p<0.001)$. Thus, degrees of

757 freedom were corrected using Greenhouse-Geisser estimates of sphericity (Duration, $\varepsilon=0.68$;

758 Experiment ${ }^{\star}$ Duration, $\varepsilon=0.77$; Duration ${ }^{\star}$ Run, $\varepsilon=0.62$; Experiment ${ }^{\star}$ Duration ${ }^{\star}$ Run, $\varepsilon=0.49$ ). We

759 observed significant effects of Duration, $F_{(3.4,173.47)}=20.11, p<0.001, \eta^{2}=0.28$, Run, $F_{(2,102)}=$ $76012.48, p<0.001, \eta^{2}=0.20$, and the interactions Duration ${ }^{\star} R u n, F_{(10,315.08)}=13.81, p<0.001, \eta^{2}=$ 7610.21 , and Duration ${ }^{*}$ Run ${ }^{*}$ Group, $F_{(12.36,315.08)}=20.11, p<0.05, \eta^{2}=0.08$. Pairwise comparisons 762 were carried out, to test whether between groups differences (SC vs. CB, CB vs. LB, SC vs. LB)

763 existed for each run and duration. No significant difference was observed between the 54 764 contrasts of interest (all $p>0.05$, corrected). Data are plotted in Figure S2B, showing similar 765 trends across groups for all durations.

766

767

Correlation between LB's performance with Onset and Duration of blindness

768 We performed linear correlations between LB participants' onset of blindness and duration of 769 blindness with (1) their overall performance in each experiment (Supplementary information, 770 Figure S5A) and (2) their performance for each duration in each experiment (Figure S5B and $771 \mathrm{~S} 5 \mathrm{C})$. Pearson's correlation coefficient (RHO) between each pair pairwise comparison was 772 computed, together with p-values. We observed no significant correlation for most of the 773 conditions and the variable tested (all $p>0.05$ ). Correlations were performed and plotted with 774 MATLAB.

\section{ACKNOWLEDGMENTS}

778

779 We want to thank Antonio Quatraro, Istituto per la Ricerca la Formazione e la Riabilitazione 780 (IRIFOR) in Florence, Unione Italiana Ciechi (UIC) in Lucca, Pisa, Pistoia, Prato, Trento, and 781 Istituto Cavazza in Bologna. Finally, we are grateful to Dr. Peter Neri for providing insights on the 782 analyses and interpretation of the results. Funding: Davide Bottari (PRIN 2017 research grant. 783 Prot. 20177894ZH) 


\section{REFERENCES}

Plomp, R. (1964). Rate of decay of auditory sensation. The Journal of the Acoustical Society of America, 36(2), 277-282.

Yabe, H., Tervaniemi, M., Sinkkonen, J., Huotilainen, M., Ilmoniemi, R. J., \& Näätänen, R. (1998). Temporal window of integration of auditory information in the human brain. Psychophysiology, 35(5), 615-619.

McDermott, J. H., Schemitsch, M., \& Simoncelli, E. P. (2013). Summary statistics in auditory perception. Nature neuroscience, 16(4), 493-498.

Barlow, H. B. (1961). Possible principles underlying the transformation of sensory messages. Sensory communication, 1, 217-234.

McDermott, J. H., \& Simoncelli, E. P. (2011). Sound texture perception via statistics of the auditory periphery: evidence from sound synthesis. Neuron, 71(5), 926-940.

Ruggero, M. A. (1992). Responses to sound of the basilar membrane of the mammalian cochlea. Current opinion in neurobiology, 2(4), 449-456.

Dau, T., Kollmeier, B., \& Kohlrausch, A. (1997). Modeling auditory processing of amplitude modulation. I. Detection and masking with narrow-band carriers. The Journal of the Acoustical Society of America, 102(5), 2892-2905.

Gygi, B., Kidd, G. R., \& Watson, C. S. (2004). Spectral-temporal factors in the identification of environmental sounds. The Journal of the Acoustical Society of America, 115(3), 1252-1265.

Joris, P. X., Schreiner, C. E., \& Rees, A. (2004). Neural processing of amplitude-modulated sounds. Physiological reviews, 84(2), 541-577.

Baumann, S., Griffiths, T. D., Sun, L., Petkov, C. I., Thiele, A., \& Rees, A. (2011). Orthogonal representation of sound dimensions in the primate midbrain. Nature neuroscience, 14(4), 423-425

McWalter, R., \& McDermott, J. H. (2018). Adaptive and selective time averaging of auditory scenes. Current Biology, 28(9), 1405-1418.

Mowery, T. M., Kotak, V. C., \& Sanes, D. H. (2016). The onset of visual experience gates auditory cortex critical periods. Nature communications, 7(1), 1-11.

Kayser, C., Petkov, C. I., \& Logothetis, N. K. (2008). Visual modulation of neurons in auditory cortex. Cerebral Cortex, 18(7), 1560-1574.

Thorne, J. D., De Vos, M., Viola, F. C., \& Debener, S. (2011). Cross-modal phase reset predicts auditory task performance in humans. Journal of Neuroscience, 31(10), 3853-3861.

Golumbic, E. Z., Cogan, G. B., Schroeder, C. E., \& Poeppel, D. (2013). Visual input enhances selective speech envelope tracking in auditory cortex at a "cocktail party". Journal of Neuroscience, 33(4), 1417-1426.

Shamma, S. (2001). On the role of space and time in auditory processing. Trends in cognitive sciences, 5(8), 340-348.

Portilla, J., \& Simoncelli, E. P. (2000). A parametric texture model based on joint statistics of complex wavelet coefficients. International journal of computer vision, 40(1), 49-70. 
Röder, B., Kekunnaya, R., \& Guerreiro, M. J. (2020). Neural mechanisms of visual sensitive periods in humans. Neuroscience \& Biobehavioral Reviews.

Pavani, F., \& Röder, B. (2012). Crossmodal plasticity as a consequence of sensory loss: insights from blindness and deafness. The new handbook of multisensory processes, 737-759.

Gori, M., Sandini, G., Martinoli, C., \& Burr, D. C. (2014). Impairment of auditory spatial localization in congenitally blind human subjects. Brain, 137(1), 288-293

Battal, C., Rezk, M., Mattioni, S., Vadlamudi, J., \& Collignon, O. (2019). Representation of auditory motion directions and sound source locations in the human planum temporale. Journal of Neuroscience, 39(12), 2208-2220.

Röder, B., \& Rösler, F. (2003). Memory for environmental sounds in sighted, congenitally blind and late blind adults: evidence for cross-modal compensation. International Journal of Psychophysiology, 50(1-2), 27-39.

Amedi, A., Raz, N., Pianka, P., Malach, R., \& Zohary, E. (2003). Early 'visual'cortex activation correlates with superior verbal memory performance in the blind. Nature neuroscience, 6(7), 758766.

Huber, E., Chang, K., Alvarez, I., Hundle, A., Bridge, H., \& Fine, I. (2019). Early blindness shapes cortical representations of auditory frequency within auditory cortex. Journal of Neuroscience, 39(26), 5143-5152.

Trouvain, J. (2007). On the comprehension of extremely fast synthetic speech.

Dietrich, S., Hertrich, I., \& Ackermann, H. (2013). Ultra-fast speech comprehension in blind subjects engages primary visual cortex, fusiform gyrus, and pulvinar-a functional magnetic resonance imaging (fMRI) study. BMC neuroscience, 14(1), 74.

Muchnik, C., Efrati, M., Nemeth, E., Malin, M., \& Hildesheimer, M. (1991). Central auditory skills in blind and sighted subjects. Scandinavian audiology, 20(1), 19-23.

Park, H., Kayser, C., Thut, G., \& Gross, J. (2016). Lip movements entrain the observers' lowfrequency brain oscillations to facilitate speech intelligibility. Elife, 5, e14521.

Rogers Montgomery, C., \& Clarkson, M. G. (1997). Infants' pitch perception: masking by low-and high-frequency noises. The Journal of the Acoustical Society of America, 102(6), 3665-3672.

Saint-Arnaud, N., \& Popat, K. (1995). Analysis and synthesis of sound textures. In in Readings in Computational Auditory Scene Analysis.

Schwarz, D. (2011, September). State of the art in sound texture synthesis.

Lorenzi, C., Berthommier, F., Apoux, F., \& Bacri, N. (1999). Effects of envelope expansion on speech recognition. Hearing research, 136(1-2), 131-138.

Bacon, S. P., \& Grantham, D. W. (1989). Modulation masking: Effects of modulation frequency, depth, and phase. The Journal of the Acoustical Society of America, 85(6), 2575-2580.

Smith, Z. M., Delgutte, B., \& Oxenham, A. J. (2002). Chimaeric sounds reveal dichotomies in auditory perception. Nature, 416(6876), 87-90.

Gori, M., Amadeo, M. B., \& Campus, C. (2020). Spatial metric in blindness: behavioural and cortical processing. Neuroscience \& Biobehavioral Reviews, 109, 54-62. 
Gori, M., Amadeo, M. B., \& Campus, C. (2020). Temporal cues trick the visual and auditory cortices mimicking spatial cues in blind individuals. Human Brain Mapping, 41(8), 2077-2091.

Doucet, M. E., Guillemot, J. P., Lassonde, M., Gagné, J. P., Leclerc, C., \& Lepore, F. (2005). Blind subjects process auditory spectral cues more efficiently than sighted individuals. Experimental brain research, 160(2), 194-202.

Röder, B., Teder-SaĖlejaÈrvi, W., Sterr, A., Rösler, F., Hillyard, S. A., \& Neville, H. J. (1999). Improved auditory spatial tuning in blind humans. Nature, 400(6740), 162-166.

Moos, A., \& Trouvain, J. (2007). Comprehension of Ultra-Fast Speech-Blind vs.'Normally Hearing'Persons. In Proceedings of the 16th International Congress of Phonetic Sciences (Vol. 1, pp. 677-680). Saarland University Saarbrücken, Germany.

Weaver, K. E., \& Stevens, A. A. (2006). Auditory gap detection in the early blind. Hearing research, 211(1-2), 1-6.

Starlinger, I., \& Niemeyer, W. (1981). Do the blind hear better? Investigations on auditory processing in congenital or early acquired blindness I. Peripheral functions. Audiology, 20(6), 503-509.

Putzar, L., Hötting, K., \& Röder, B. (2010). Early visual deprivation affects the development of face recognition and of audio-visualaudio-visual speech perception. Restorative neurology and neuroscience, 28(2), 251-257.

Chi, T., Ru, P., \& Shamma, S. A. (2005). Multiresolution spectrotemporal analysis of complex sounds. The Journal of the Acoustical Society of America, 118(2), 887-906.

Norman-Haignere, S. V., \& McDermott, J. H. (2018). Neural responses to natural and modelmatched stimuli reveal distinct computations in primary and nonprimary auditory cortex. PLoS biology, 16(12), e2005127.

Maxon, A. B., \& Hochberg, I. (1982). Development of psychoacoustic behavior: Sensitivity and discrimination. Ear and Hearing, 3(6), 301-308.

Jensen, J. K., \& Neff, D. L. (1993). Development of basic auditory discrimination in preschool children. Psychological Science, 4(2), 104-107.

Moore, D. R., Cowan, J. A., Riley, A., Edmondson-Jones, A. M., \& Ferguson, M. A. (2011). Development of auditory processing in 6-to 11-yr-old children. Ear and hearing, 32(3), 269-285.

Banai, K., Sabin, A. T., \& Wright, B. A. (2011). Separable developmental trajectories for the abilities to detect auditory amplitude and frequency modulation. Hearing research, 280(1-2), 219227.

Gori, M., Del Viva, M., Sandini, G., \& Burr, D. C. (2008). Young children do not integrate visual and haptic form information. Current Biology, 18(9), 694-698.

Brandwein, A. B., Foxe, J. J., Russo, N. N., Altschuler, T. S., Gomes, H., \& Molholm, S. (2011). The development of audio-visualaudiovisual multisensory integration across childhood and early adolescence: a high-density electrical mapping study. Cerebral Cortex, 21(5), 1042-1055.

Ross, L. A., Molholm, S., Blanco, D., Gomez-Ramirez, M., Saint-Amour, D., \& Foxe, J. J. (2011). The development of multisensory speech perception continues into the late childhood years. European Journal of Neuroscience, 33(12), 2329-2337. 
Lyon, R., \& Shamma, S. (1996). Auditory representations of timbre and pitch. In Auditory computation (pp. 221-270). Springer, New York, NY.

Shamma, S. A. (1985). Speech processing in the auditory system II: Lateral inhibition and the central processing of speech evoked activity in the auditory nerve. The Journal of the Acoustical Society of America, 78(5), 1622-1632.

Watanabe, T., \& Sasaki, Y. (2015). Perceptual learning: toward a comprehensive theory. Annual review of psychology, 66, 197-221.

de Villers-Sidani, E., \& Merzenich, M. M. (2011). Lifelong plasticity in the rat auditory cortex: basic mechanisms and role of sensory experience. In Progress in brain research (Vol. 191, pp. 119131). Elsevier.

Picard, D., Dacremont, C., Valentin, D., \& Giboreau, A. (2003). Perceptual dimensions of tactile textures. Acta psychologica, 114(2), 165-184.

Weber, A. I., Saal, H. P., Lieber, J. D., Cheng, J. W., Manfredi, L. R., Dammann, J. F., \& Bensmaia, S. J. (2013). Spatial and temporal codes mediate the tactile perception of natural textures. Proceedings of the National Academy of Sciences, 110(42), 17107-17112.

Cramer, A. O., van Ravenzwaaij, D., Matzke, D., Steingroever, H., Wetzels, R., Grasman, R. P., ... \& Wagenmakers, E. J. (2016). Hidden multiplicity in exploratory multiway ANOVA: Prevalence and remedies. Psychonomic bulletin \& review, 23(2), 640-647.

Benjamini, Y., Drai, D., Elmer, G., Kafkafi, N., \& Golani, I. (2001). Controlling the false discovery rate in behavior genetics research. Behavioural brain research, 125(1-2), 279-284 
Table 1. Characteristics of blind participants.

1. Late-onset blinds group $(\mathrm{N}=18)$

\begin{tabular}{|c|c|c|c|c|c|c|c|c|}
\hline & Age & Sex & Hand & Residual & $\begin{array}{l}\text { Onset } \\
\text { (years) }\end{array}$ & Etiology & Education & Music \\
\hline LB01 & 21 & M & $\mathrm{R}$ & None & 18 & $\begin{array}{l}\text { Congenital } \\
\text { Glaucoma }\end{array}$ & $\begin{array}{l}\text { Middle } \\
\text { School }\end{array}$ & No \\
\hline LB02 & 26 & M & $\mathrm{R}$ & LP & 18 & $\begin{array}{l}\text { Retinitis } \\
\text { pigmentosa }\end{array}$ & University & No \\
\hline LB03 & 44 & $\mathrm{~F}$ & $\mathrm{R}$ & LP & 20 & $\begin{array}{l}\text { Retinitis } \\
\text { pigmentosa }\end{array}$ & High School & No \\
\hline LB04 & 25 & $\mathrm{~F}$ & $\mathrm{R}$ & None & $\begin{array}{l}22 \\
\text { LE } 14 \\
\text { RE } 22\end{array}$ & $\begin{array}{l}\text { Stevens- } \\
\text { Johnson } \\
\text { syndrome }\end{array}$ & University & No \\
\hline LB05 & 41 & $M$ & $\mathrm{R}$ & None & 36 & $\begin{array}{l}\text { Familial } \\
\text { exudative } \\
\text { vitreoretinopathy }\end{array}$ & High School & No \\
\hline LB06 & 30 & $\mathrm{~F}$ & $\mathrm{R}$ & None & 20 & Eye tumor & High School & No \\
\hline LB07 & 38 & $\mathrm{~F}$ & $\mathrm{R}$ & None & 15 & Glaucoma & University & No \\
\hline LB08 & 59 & M & $\mathrm{R}$ & None & 43 & $\begin{array}{l}\text { Optic Nerve } \\
\text { Sheath } \\
\text { Meningioma }\end{array}$ & $\begin{array}{l}\text { Middle } \\
\text { School }\end{array}$ & No \\
\hline LB09 & 55 & $F$ & $\mathrm{R}$ & LP & 30 & $\begin{array}{l}\text { Retinitis } \\
\text { pigmentosa }\end{array}$ & University & No \\
\hline LB10 & 52 & $\mathrm{~F}$ & $\mathrm{R}$ & None & 27 & $\begin{array}{l}\text { Retinitis } \\
\text { pigmentosa }\end{array}$ & High School & No \\
\hline LB11 & 55 & $F$ & $\mathrm{R}$ & $\begin{array}{l}\text { LP, } \quad \text { SP, } \\
\text { MP }\end{array}$ & 51 & $\begin{array}{l}\text { Bietti's } \\
\text { Crystalline } \\
\text { Dystrophy }\end{array}$ & University & No \\
\hline LB12 & 46 & M & $\mathrm{R}$ & LP & 18 & $\begin{array}{l}\text { 6mo: Removed } \\
\text { congenital } \\
\text { cataract; then } \\
\text { Glaucoma }\end{array}$ & $\begin{array}{l}\text { Middle } \\
\text { School }\end{array}$ & No \\
\hline LB13 & 50 & $\mathrm{~F}$ & $\mathrm{R}$ & LP & 11 & $\begin{array}{l}\text { Retinitis } \\
\text { pigmentosa }\end{array}$ & High School & No \\
\hline LB14 & 55 & $M$ & $\mathrm{R}$ & LP & 38 & $\begin{array}{l}\text { Retinitis } \\
\text { pigmentosa }\end{array}$ & $\begin{array}{l}\text { Middle } \\
\text { School }\end{array}$ & No \\
\hline LB15 & 40 & $\mathrm{~F}$ & $\mathrm{R}$ & None & 20 & Glaucoma & High School & No \\
\hline
\end{tabular}




\begin{tabular}{|l|l|l|l|l|l|l|l|l|}
\hline LB16 & 32 & M & R & LP & 10 & $\begin{array}{l}\text { Optic nerve } \\
\text { compression } \\
\text { Astrocytoma }\end{array}$ & University & No \\
\hline LB17 & 22 & M & R & None & 20 & Glaucoma & High School & No \\
\hline LB18 & 30 & M & R & LP in RE & 18 & $\begin{array}{l}\text { Glaucoma (RE) } \\
\text { and Retinal } \\
\text { detachment (LE) }\end{array}$ & High School & No \\
\hline
\end{tabular}

2. Congenitally blind group $(\mathrm{N}=18)$

\begin{tabular}{|c|c|c|c|c|c|c|c|c|}
\hline CB01 & 32 & $\mathrm{M}$ & $\mathrm{R}$ & LP & 0 & $\begin{array}{l}\text { Congenital } \\
\text { Glaucoma }\end{array}$ & University & No \\
\hline CB02 & 36 & M & $\mathrm{R}$ & None & 0 & $\begin{array}{l}\text { Retinitis } \\
\text { pigmentosa }\end{array}$ & High School & No \\
\hline CB03 & 44 & M & $\mathrm{R}$ & None & 0 & $\begin{array}{l}\text { Congenital } \\
\text { Glaucoma }\end{array}$ & High School & No \\
\hline CB04 & 60 & $\mathrm{~F}$ & $\mathrm{R}$ & LP & 0 & $\begin{array}{l}\text { Retinopathy of } \\
\text { prematurity }\end{array}$ & High School & Yes \\
\hline CB05 & 45 & M & $\mathrm{R}$ & LP & 0 & $\begin{array}{l}\text { Congenital } \\
\text { Glaucoma }\end{array}$ & University & No \\
\hline CB06 & 43 & $\mathrm{~F}$ & $\mathrm{R}$ & None & 0 & $\begin{array}{l}\text { Retinopathy of } \\
\text { prematurity }\end{array}$ & High School & No \\
\hline CB07 & 28 & $\mathrm{~F}$ & $\mathrm{R}$ & LP & 0 & Microphthalmia & University & No \\
\hline CB08 & 29 & $F$ & L & None & 0 & $\begin{array}{l}\text { Retinopathy of } \\
\text { prematurity }\end{array}$ & University & No \\
\hline CB09 & 27 & M & $\mathrm{R}$ & LP & 0 & $\begin{array}{l}\text { Retinopathy of } \\
\text { prematurity }\end{array}$ & High School & Yes \\
\hline CB10 & 41 & M & $\mathrm{R}$ & LP & 0 & $\begin{array}{l}\text { Retinitis } \\
\text { pigmentosa }\end{array}$ & University & No \\
\hline CB11 & 59 & M & $\mathrm{R}$ & None & 0 & Glaucoma & High School & Yes \\
\hline CB12 & 37 & $\mathrm{~F}$ & $R / L$ & LP & 0 & $\begin{array}{l}\text { Congenital } \\
\text { cataract }\end{array}$ & High School & No \\
\hline CB13 & 20 & $\mathrm{~F}$ & $\mathrm{R}$ & None & 0 & $\begin{array}{l}\text { Microphthalmia } \\
\text { and Aniridia }\end{array}$ & University & No \\
\hline CB14 & 34 & $\mathrm{~F}$ & $\mathrm{R}$ & LP & 0 & $\begin{array}{l}\text { Optic nerve } \\
\text { hypoplasia }\end{array}$ & University & No \\
\hline CB15 & 32 & M & $\mathrm{R}$ & LP & 0 & $\begin{array}{l}\text { Retinopathy of } \\
\text { prematurity }\end{array}$ & University & Yes \\
\hline CB16 & 30 & M & $\mathrm{R}$ & LP & 0 & $\begin{array}{l}\text { Leber's } \\
\text { congenital }\end{array}$ & University & Yes \\
\hline
\end{tabular}


bioRxiv preprint doi: https://doi.org/10.1101/2020.12.16.423049; this version posted June 1, 2021. The copyright holder for this preprint (which was not certified by peer review) is the author/funder, who has granted bioRxiv a license to display the preprint in perpetuity. It is made available under aCC-BY-NC-ND 4.0 International license.

\begin{tabular}{|l|l|l|l|l|l|l|l|l|l|}
\hline & & & & & & amaurosis & \\
\hline CB17 & 44 & F & R & None & 0 & $\begin{array}{l}\text { Virus during } \\
\text { pregnancy }\end{array}$ & High School & No \\
\hline CB18 & 28 & F & R & None & 0 & $\begin{array}{l}\text { Retinopathy of } \\
\text { prematurity }\end{array}$ & University & No \\
\hline
\end{tabular}

$\mathrm{LP}=$ light perception; $\mathrm{SP}=$ Silhouette perception; $\mathrm{MP}=$ motion perception; $\mathrm{M}=$ male; $\mathrm{F}=$ female; $\mathrm{mo}=$ month old; $\mathrm{LE}=$ left eye; $\mathrm{RE}=$ right eye; Music Training: Yes = Professional, studied music for at least 
bioRxiv preprint doi: https://doi.org/10.1101/2020.12.16.423049; this version posted June 1, 2021. The copyright holder for this preprint (which was not certified by peer review) is the author/funder, who has granted bioRxiv a license to display the preprint in perpetuity. It is made available under aCC-BY-NC-ND 4.0 International license.

785 
bioRxiv preprint doi: https://doi.org/10.1101/2020.12.16.423049; this version posted June 1, 2021. The copyright holder for this preprint (which was not certified by peer review) is the author/funder, who has granted bioRxiv a license to display the preprint in perpetuity. It is made available under aCC-BY-NC-ND 4.0 International license.

786 
bioRxiv preprint doi: https://doi.org/10.1101/2020.12.16.423049; this version posted June 1, 2021. The copyright holder for this preprint (which was not certified by peer review) is the author/funder, who has granted bioRxiv a license to display the preprint in perpetuity. It is made available under aCC-BY-NC-ND 4.0 International license. 
bioRxiv preprint doi: https://doi.org/10.1101/2020.12.16.423049; this version posted June 1, 2021. The copyright holder for this preprint (which was not certified by peer review) is the author/funder, who has granted bioRxiv a license to display the preprint in perpetuity. It is made available under aCC-BY-NC-ND 4.0 International license.

788 
bioRxiv preprint doi: https://doi.org/10.1101/2020.12.16.423049; this version posted June 1, 2021. The copyright holder for this preprint (which was not certified by peer review) is the author/funder, who has granted bioRxiv a license to display the preprint in perpetuity. It is made available under aCC-BY-NC-ND 4.0 International license. 